\title{
Benchmarking of 3D space charge codes using direct phase space measurements from photoemission high voltage dc gun
}

\author{
Ivan V. Bazarov,* Bruce M. Dunham, Colwyn Gulliford, Yulin Li, Xianghong Liu, Charles K. Sinclair, and Ken Soong \\ Laboratory for Elementary Particle Physics, Cornell University, Ithaca, New York 14853, USA \\ Fay Hannon \\ Lancaster University, Lancaster, United Kingdom \\ (Received 3 July 2008; published 30 October 2008)
}

\begin{abstract}
We present a comparison between space charge calculations and direct measurements of the transverse phase space of space charge dominated electron bunches from a high voltage dc photoemission gun followed by an emittance compensation solenoid magnet. The measurements were performed using a double-slit emittance measurement system over a range of bunch charge and solenoid current values. The data are compared with detailed simulations using the 3D space charge codes GPT and PARMELA3D. The initial particle distributions were generated from measured transverse and temporal laser beam profiles at the photocathode. The beam brightness as a function of beam fraction is calculated for the measured phase space maps and found to approach within a factor of 2 the theoretical maximum set by the thermal energy and the accelerating field at the photocathode.
\end{abstract}

DOI: 10.1103/PhysRevSTAB.11.100703

PACS numbers: 29.25.Bx, 29.20.Ej, 29.27.Bd

\section{INTRODUCTION}

The generation of high-brightness electron beams remains the principal challenge for a number of linear accelerator based projects, including the Energy Recovery Linac (ERL) synchrotron light source under development at Cornell. The design of electron injectors for these machines relies heavily on the use of space charge simulations [1-6]. There are a number of open questions with regard to understanding and modeling the evolution of space charge dominated bunched beams from photoemission guns. Different space charge codes use different approximations in their attempt to capture the most significant physics relevant to beam dynamics in photoinjectors. Certain codes allow self-consistent inclusion of complex conducting boundaries at the expense of a considerable increase in the required computation time $[7,8]$, while many widely used codes assume open boundary conditions everywhere except in the vicinity of the photocathode. Since it is not possible to use direct self-force calculations for a bunch containing $\sim 10^{9}$ particles, macroparticles have to be used to represent the actual bunch. Artificial smoothing of the space charge potential is employed either through meshing of the electron cloud or by introduction of an effective size to the macroparticles in a point-to-point calculation. As a result, the space charge forces in a simulation may be either overly smooth or grainy compared to the actual self-forces in the bunch. Additional assumptions are employed by different codes regarding the details of emission from the photocathode, whether or not the velocities of individual

\footnotetext{
*ib38@ cornell.edu
}

electrons in the rest frame of the bunch can be treated as negligible, the assumed distribution of the space charge (e.g. uniformly populated cylinders in HOMDYN [4]), etc. The validity of these assumptions must be evaluated for each individual case. Unfortunately, data comparing directly measured beam phase space distributions with simulations are sparse for space charge dominated bunched beams such as those found in dc and rf photoinjectors [9-11]. Simulation of even a relatively simple configuration involves a number of "free" parameters that must be varied within the uncertainty of their measurement, such as the $\mathrm{rf}$ phase in an $\mathrm{rf}$ gun, to obtain good agreement between simulations and measurements [11].

We present direct measurements of the transverse phase space distribution using a simple beam line consisting of a dc gun followed by an emittance compensation solenoid. The number of variables affecting the beam quality is reduced to a minimum in this setup. Careful characterization of the initial conditions, such as the laser beam transverse and temporal profiles and the thermal emittance of the photocathode, allows us to carry out a direct crosscheck between the measurements and 3D space charge simulations using the PARMELA3D [1] and GPT [3] codes. Finally, the measured phase space distributions are used to calculate the beam brightness as a function of the included beam fraction, which is compared to the theoretical limit set by the cathode transverse thermal energy and the accelerating field at the photocathode.

Section II of this paper gives details of our experimental setup, beam diagnostics, and experimental procedures. Section III presents details on the simulations, and data processing techniques used to extract information such as 
second moments and rms emittances from the measurements. A comparison between the processed data and our simulations follows in Sec. IV. Finally, we conclude with a discussion and outlook for future work.

\section{EXPERIMENTAL SETUP}

\section{A. Beam line}

Figure 1 shows the experimental setup, which consists of a high voltage dc gun [12] followed by an emittance compensation solenoid $0.335 \mathrm{~m}$ from the photocathode. The high voltage (HV) dc gun, designed for a maximum of $750 \mathrm{kV}$, was operated at $250 \mathrm{kV}$ for these measurements. The electric field profile along the electrostatic axis of the gun is shown in Fig. 2(a). The gun reached $420 \mathrm{kV}$ during high voltage processing in 2007. Since then, however, we had to limit the gun voltage to a conservative value below $300 \mathrm{kV}$ due to field emission problems. Subsequent dis- assembly of the gun revealed a considerable quantity of dust originating from the resistive coating of the ceramic. This is believed to be the primary reason behind the strong field emission; work is underway to eliminate this source of dust from the gun.

Figure 2(b) shows the magnetic field profile of the emittance compensation solenoid as calculated by POISSON [13] and as measured with a Hall-probe. A double-slit emittance measurement system (EMS) and an insertable viewscreen are positioned $1.244 \mathrm{~m}$ from the photocathode. The beam line also includes a deflecting $\mathrm{rf}$ cavity [14] for measurement of the initial temporal profile of the photoemitted electron bunches, two magnetic beam scanners, and a Faraday cup, all integrated into the data acquisition system. The EMS is described in the next subsection. Two different materials are employed for viewscreens: high sensitivity $\mathrm{BeO}$ used in temporal measurements with very low bunch charges, and a chemical vapor

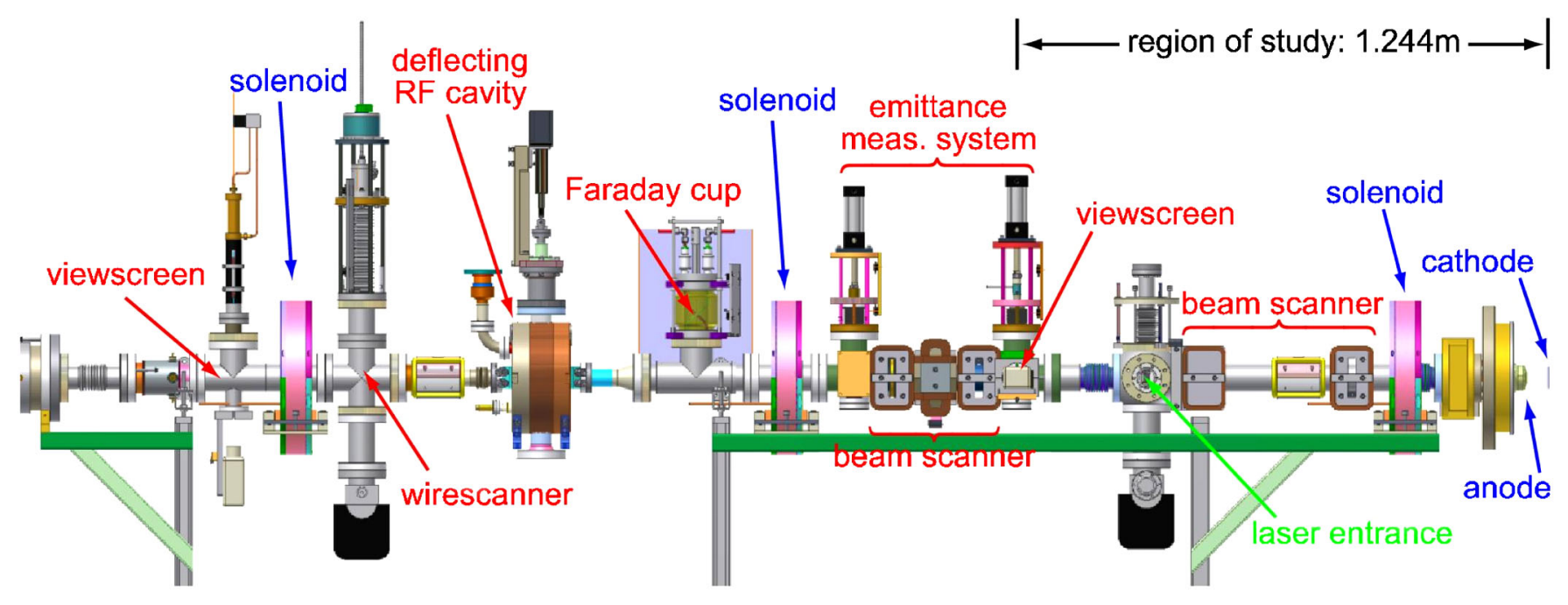

FIG. 1. (Color) The beam line used in the space charge studies. Beam direction is to the left.
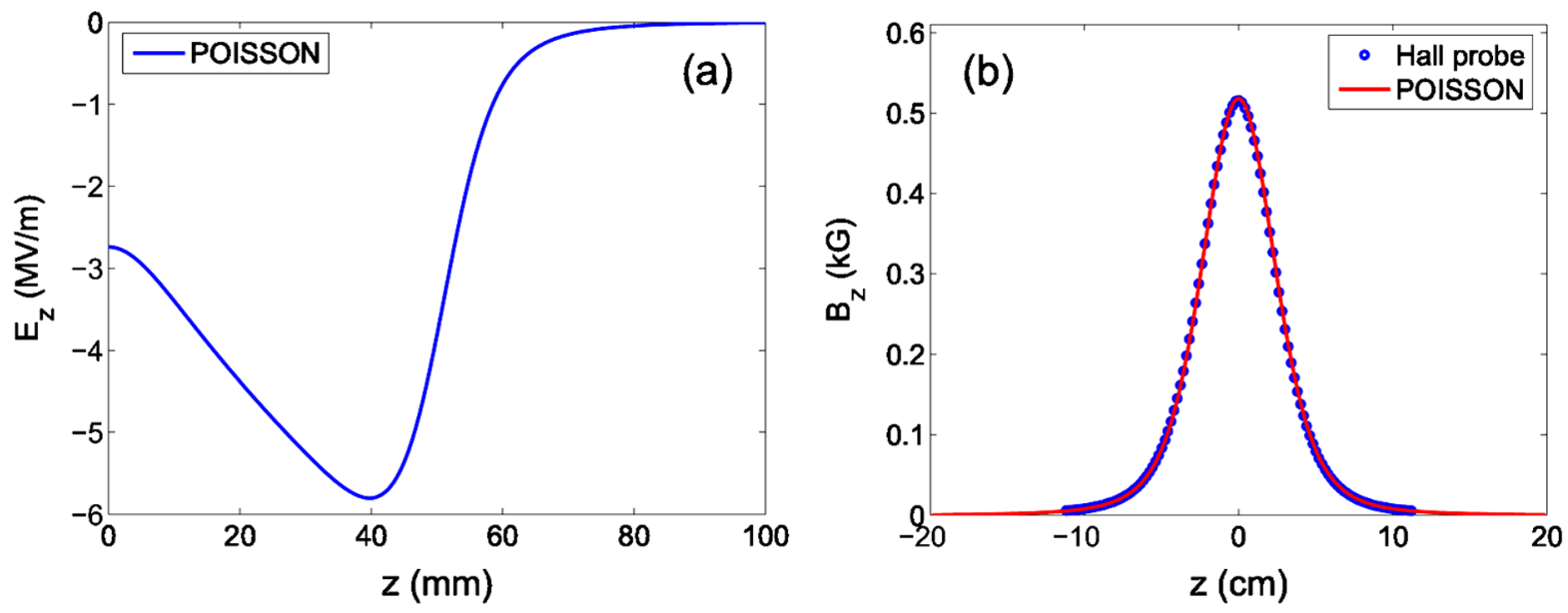

FIG. 2. (Color) Field profiles for (a) the dc gun at $250 \mathrm{kV}$ and (b) the solenoid with 5 A excitation current. 
deposition diamond used with average beam currents up to $100 \mu \mathrm{A}$. Each viewscreen is viewed by a 12-bit CCD camera interfaced to the data acquisition system.

The laser system has been detailed elsewhere [15]. It produces a $50 \mathrm{MHz}$ train of pulses with an average power of about 1 Watt at a wavelength of $520 \mathrm{~nm}$. The laser spotsize was monitored with a 12-bit CCD camera at the location of a virtual cathode. A Pockels cell was used to chop the $50 \mathrm{MHz}$ train of pulses to a much lower duty factor for beam measurements. Typical average beam currents during phase space measurements were between 10 and $100 \mu \mathrm{A}$.

\section{B. Emittance measurement system}

Our EMS is a double-slit system, with no moving parts, as shown in Fig. 3. Two magnetic beam scanners, one before the 1st slit, and one between the two slits, are used to sweep the beam across the slits. Each scanner has two pairs of identical air core coils, with equal and opposite excitation in each pair. Each coil produces a field with negligible sextupole component across most of the beam pipe cross section, providing a uniform kick to the beam passing through it, as shown in Fig. 4. The coil pairs in each beam scanner have been measured to cancel each other to better than $1 \%$. Thus, each scanner changes only the position of the beam at the slit following it without affecting its divergence. In addition, a pair of horizontal and vertical steering coils before each of the slits allows correction for yaw/pitch alignment errors, leading to overall relaxed tolerances for the system. Finally, a weak solenoid (providing $\pm 1^{\circ}$ of beam rotation) is positioned between the two slits to allow for roll compensation, although this proved unnecessary in practice.

The 1st EMS slit consists of a horizontal armor slit with a $200 \mu \mathrm{m}$ opening followed by a $20 \mu \mathrm{m}$ precision slit brazed to the water-cooled armor slit as shown in Fig. 3

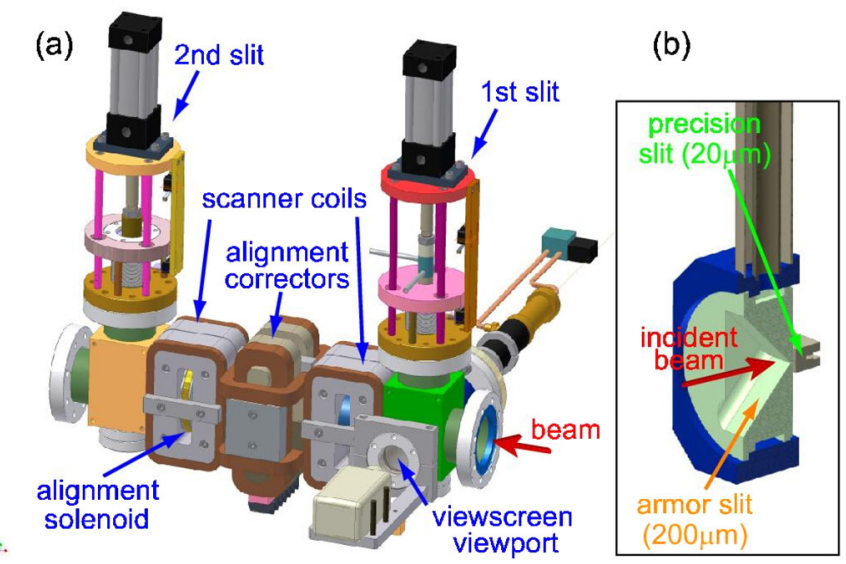

FIG. 3. (Color) (a) The emittance measurement system. A beam scanner (not shown) precedes the 1st slit. (b) Details of the 1st slit showing water-cooled $200 \mu \mathrm{m}$ armor slit and $20 \mu \mathrm{m}$ precision slit.

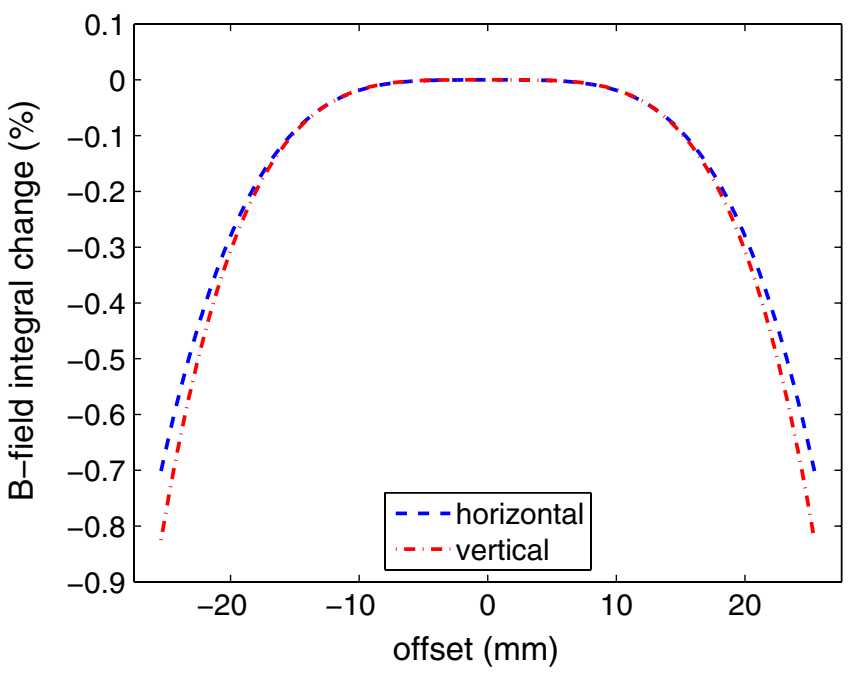

FIG. 4. (Color) Uniformity of the B-field integral for beam scanner coils. The quantity plotted is $I(x, 0) / I(0,0)-1$ and $I(0, y) / I(0,0)-1$, where $\mathcal{I}(x, y) \equiv \int B_{x}(x, y, z) d z$ and $x=$ $y=0$ offset corresponds to the center of the beam pipe.

(b). Most of the beam power is intercepted by the armor slit. ANSYS analysis shows that for the beam size used in these measurements, there is no significant deformation of the precision slit $(<10 \%)$ with $1 \mathrm{~kW}$ of incident beam power.

Phase space measurements can be carried out using either single slit followed by a viewscreen, or the doubleslit EMS system followed by a Faraday. A Monte Carlo analysis using GEANT4 [16] has demonstrated that scattered radiation from the slits does not degrade the emittance measurements for either of the two configurations [17].

All of the measurements reported in this work have been done using a double-slit method. The beam passing both slits was detected using the Faraday cup connected to a low noise current amplifier. A solenoid before the Faraday cup focused the beamlet to the center of the cup for optimum charge collection efficiency. The maximum scan rate for the beam scanners was $200 \mathrm{~Hz}$. Typical transverse phase space scans of $100 \times 100$ steps required on the order of 1 min.

Considerable care is required when designing a two-slit system for direct measurement of the phase space of a space charge dominated beam $[18,19]$. The simple requirement that the beamlet after the 1st slit be emittance dominated [18] is necessary but not sufficient. Because the ratio of the space charge and emittance terms scales essentially as $\propto \sigma_{y}^{3}$, meeting the condition at the location of the 1 st slit does not ensure that the beamlet stays emittance dominated all the way to the 2 nd slit.

We determined the slit opening size and the distance between the two slits by solving the coupled beam envelope equations including the space charge force for a beamlet selected by the 1 st slit: 


$$
\left\{\begin{array}{l}
\sigma_{x}^{\prime \prime}-\frac{I}{I_{0}(\beta \gamma)^{3}\left(\sigma_{x}+\sigma_{y}\right)}-\frac{\epsilon_{n, x}^{2}}{\sigma_{x}^{3}(\beta \gamma)^{2}}=0, \\
\sigma_{y}^{\prime \prime}-\frac{I}{I_{0}(\beta \gamma)^{3}\left(\sigma_{x}+\sigma_{y}\right)}-\frac{\epsilon_{n, y}^{2}}{\sigma_{y}^{3}(\beta \gamma)^{2}}=0 .
\end{array}\right.
$$

Here $I$ is the beam peak current after passing through the 1st slit, $I_{0}=17 \mathrm{kA}$ is the Alfvén current, and $(\beta \gamma)$ is the normalized momentum. For a horizontal slit with opening $d$ small compared to the beam size, one has $\sigma_{y}=d / \sqrt{12}$ and normalized rms emittance $\epsilon_{n, y}=\epsilon_{n, y 0}\left(d / \sqrt{12} \sigma_{y 0}\right)$, where $\epsilon_{n, y 0}$ and $\sigma_{y 0}$ are the emittance and vertical size of the full beam prior to the slit. By solving Eq. (1) for the beamlet size $\sigma_{y}(L)$ at the location $L$ of the 2 nd slit (or the viewscreen), and comparing $\sigma_{y}(L) / L$ to the uncorrelated divergence $\sigma_{y 0}^{\prime}$ at the 1 st slit position, one can gauge the effectiveness of the slit system in the presence of space charge. Figure 5 shows the results of emittance overestimation by the double-slit method in the case of $80 \mathrm{pC}$ bunches with the parameters as indicated in the figure. The separation between the two slits was chosen to be $38 \mathrm{~cm}$, leading to the measured emittance overestimating the true emittance by less than $10 \%$ at $0.3 \mu \mathrm{m}$ normalized rms emittance and $0.5 \mathrm{MeV}$ kinetic energy. For $0.25 \mathrm{MeV}$ kinetic energy with our parameters, the maximum error to emittance due to space charge between the slits is below $5 \%$ of $1.8 \mu \mathrm{m}$ normalized $\mathrm{rms}$ emittance for the $80 \mathrm{pC} /$ bunch case.

\section{Experimental procedures}

Measurements were taken at three different bunch charges: 80,20 , and $0.5 \mathrm{pC}$. The measured laser intensity

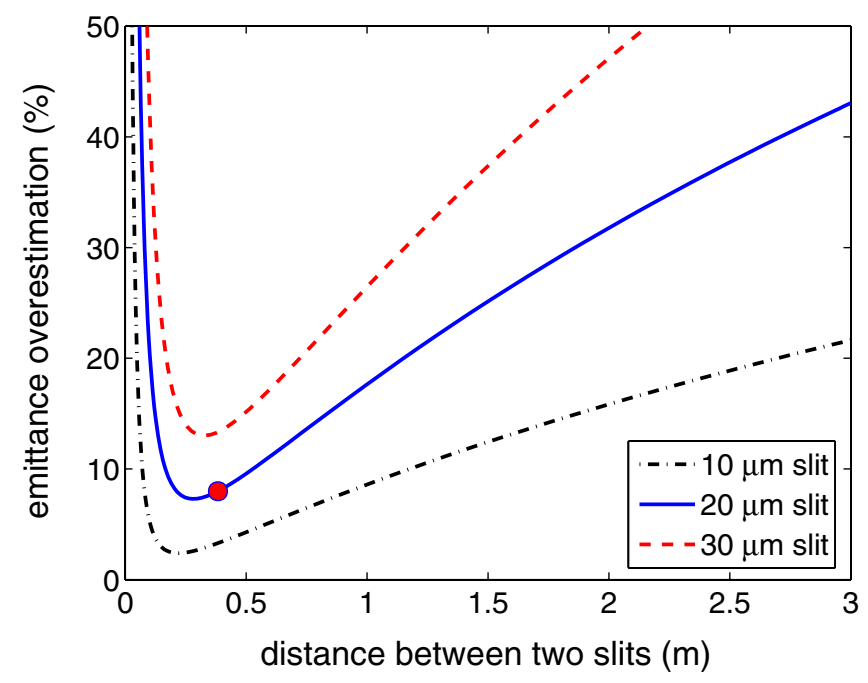

FIG. 5. (Color) Emittance overestimation due to space charge for different slit openings. Beam parameters: $\epsilon_{n, x, y}=0.3 \mu \mathrm{m}$, rms bunch duration $\sigma_{t}=20 \mathrm{ps}, \sigma_{x, y}=1.3 \mathrm{~mm}$, total rms divergence $\sigma_{T, x, y}^{\prime}=0.72 \mathrm{mrad}$, kinetic energy $0.5 \mathrm{MeV}$, and bunch charge $80 \mathrm{pC}$. The red dot shows the actual separation between the two slits $(38 \mathrm{~cm})$.

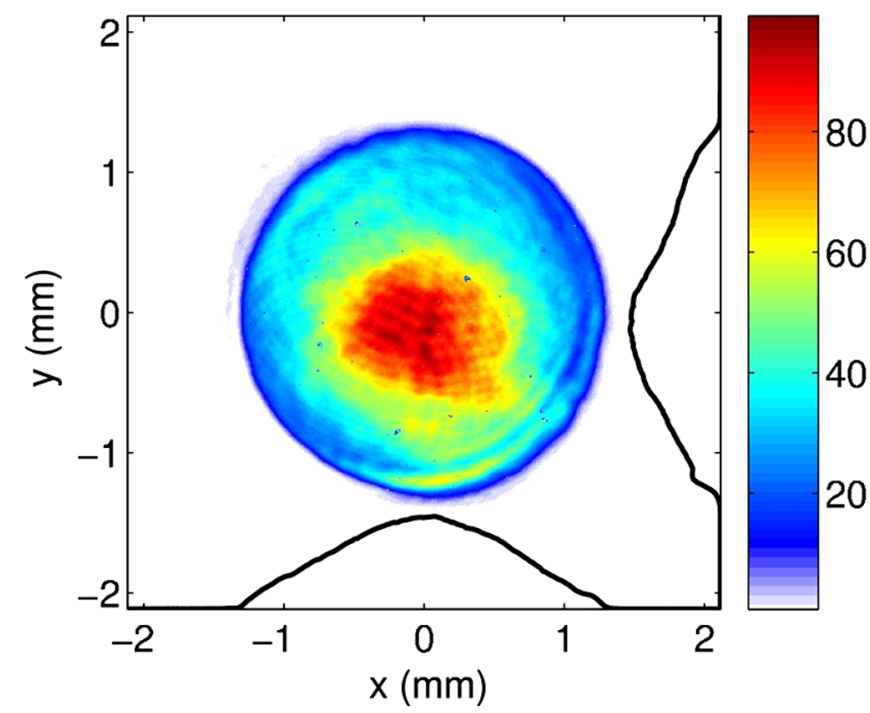

FIG. 6. (Color) A typical transverse laser profile. The black solid lines show the projected horizontal and vertical profiles.

stability was $2 \%$ rms. The laser spot was initially magnified and passed through a $2.6 \mathrm{~mm}$ diameter aperture, which was 1:1 imaged onto the photocathode. The laser position stability was $60 \mu \mathrm{m}$ rms in each transverse direction at the location of the aperture. Multiple images of the laser spot on the virtual photocathode were taken as part of each data set. An image with the centroid closest to the averaged position was chosen for use in the simulations detailed in the next section. A typical transverse laser spot profile is shown in Fig. 6.

The fundamental laser pulse was temporally divided and stacked using three birefringent crystals to produce a longer pulse with the rise time of the fundamental pulse [20]. A direct measurement of the temporal distribution of the bunch was done using the deflecting cavity and negligible charge per bunch, as shown in Fig. 7. The resolution of the temporal measurement in this case is $1.5 \mathrm{ps} \mathrm{rms}$, limited by the rf to laser synchronization and finite electron beam spot size. To obtain the actual temporal profile of the electron distribution, the data was fitted with eight variable amplitude, fixed width Gaussians, with each Gaussian assigned a $1.0 \mathrm{ps}$ sigma corresponding to the value determined from an autocorrelation measurement of the fundamental laser pulse [15]. Both the fit to the data and the reconstructed temporal profile used in the simulations are shown in Fig. 7.

Early on in the measurements, we observed asymmetric transverse phase space distributions, as shown in Fig. 18. To eliminate possible causes for this, careful beam based alignment was carried out before acquiring each data set. To do this, a small laser aperture $(0.25 \mathrm{~mm}$ diameter $)$ was placed concentric with the larger one to create a small beam with negligible charge per bunch. The center of the aperture was imaged to place the beam at the electrostatic center of the gun to within about $10 \mu \mathrm{m}$. Measurements of 


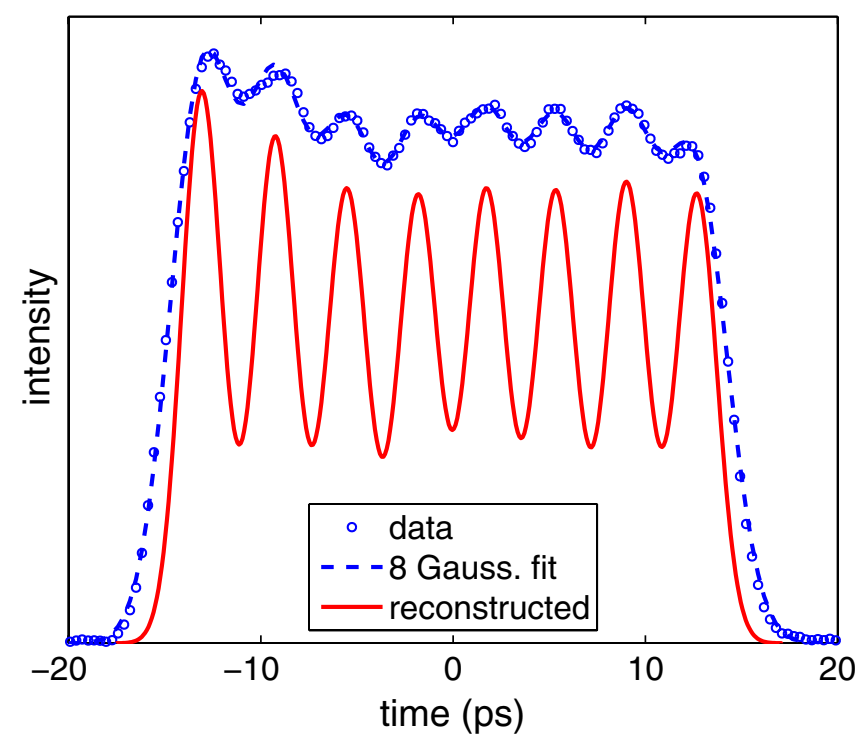

FIG. 7. (Color) The temporal profile of the electron bunches as measured by the deflecting cavity and negligible charge per bunch. The red solid line shows the reconstructed profile used in the simulations. Refer to the text for details.

the beam centroid vs the solenoid current allowed determination of the angle and offset of the magnetic axis of the solenoid with respect to the beam. The solenoid was then physically adjusted so its axis coincided with the beam axis to within a few 10's of $\mu \mathrm{m}$ and $\mu \mathrm{rad}$ in offset and angle, respectively, ensuring that the central orbit is well aligned throughout the system.

\section{SIMULATION PARAMETERS AND DATA PROCESSING PROCEDURES}

\section{A. Simulation parameters and conditions}

As the quantum efficiency (QE) of the photocathode across the region of interest was found to be uniform to better than 10\% (in absolute terms the QE for the GaAs photocathode was about $6 \%$ in this work), we have used the measured laser transverse profiles such as the one shown in Fig. 6 and the reconstructed temporal shape shown in Fig. 7 to create $3 \mathrm{D}$ distributions from the photocathode for the simulations. The thermal emittance of GaAs photocathodes has been investigated previously [21] and for an rms laser spot size $\sigma_{\perp}$ was found to be $\epsilon_{n, \text { th }}=\sigma_{\perp} \sqrt{k T_{\perp} / m c^{2}}$ with $k T_{\perp}=120 \pm 8 \mathrm{meV}$ for $520 \mathrm{~nm}$ light, $m c^{2}$ is electron rest energy.

We have used two 3D space charge codes: PARMELA3D which employs the fast Fourier transform method for solving the Poisson equation on a 3D grid [22], and general particle tracer (GPT) with a nonequidistant mesh solver for the space charge force calculation [23]. Both codes had identical field maps for the gun and the solenoid magnet. Convergence of the calculation results has been checked for 20,100 , and $500 \mathrm{k}$ particle distributions and different

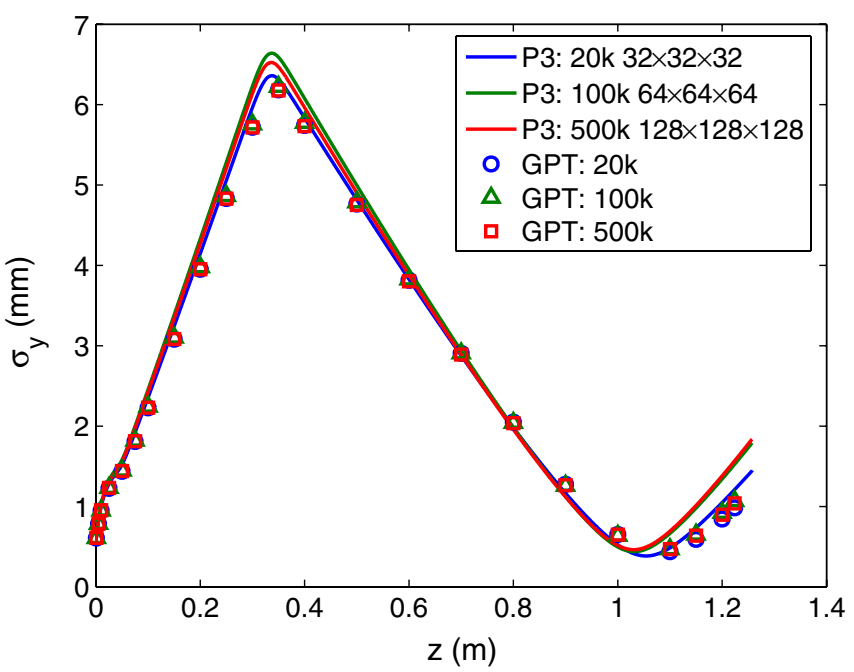

FIG. 8. (Color) Comparison of beam envelope vs longitudinal position calculations for PARMELA3D (solid lines) and GPT (open symbols) for $80 \mathrm{pC}$ charge per bunch. The solenoid current is $3.6 \mathrm{~A}$ and the gun voltage is $250 \mathrm{kV}$.

mesh sizes. The results are presented in Fig. 8 for the beam envelope and Fig. 9 for the emittance vs longitudinal position for the case of $80 \mathrm{pC}$ bunches. Only the results for the vertical plane are shown. The horizontal beam size and rms normalized emittance (not shown) differ by maximum $12 \%$ and $16 \%$ from their corresponding vertical values. An additional difference between PARMELA3D and GPT is that the former reports the relevant beam parameters as a function of time, while for the latter we have used the $3 \mathrm{D}$ coordinates of the bunch projected to a given longitudinal position. We observe that sufficient convergence is demonstrated with $100 \mathrm{k}$ macroparticles for both

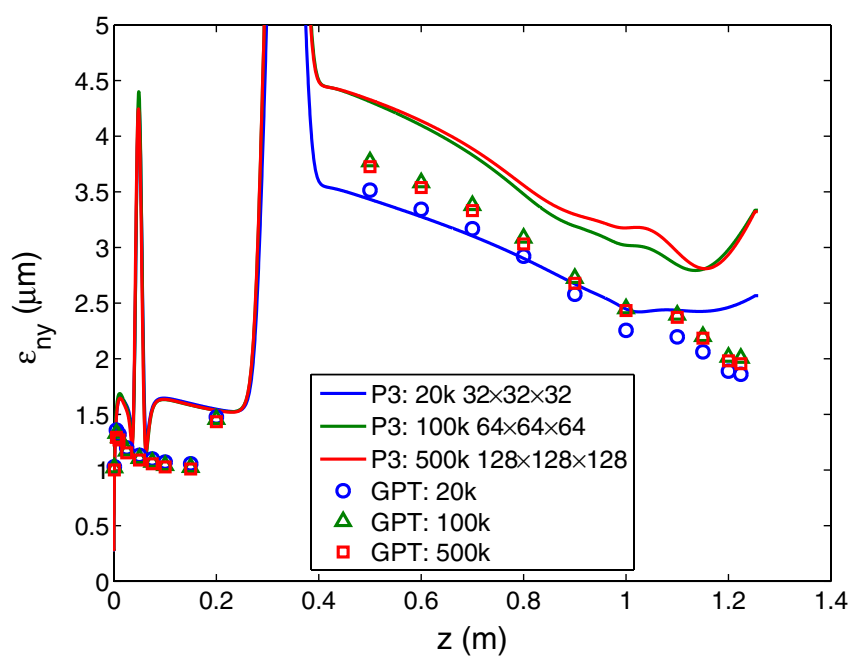

FIG. 9. (Color) Comparison of emittance vs longitudinal position calculations for PARMELA3D (solid lines) and GPT (open symbols) for $80 \mathrm{pC}$ charge per bunch. The solenoid current is $3.6 \mathrm{~A}$ and the gun voltage is $250 \mathrm{kV}$. 


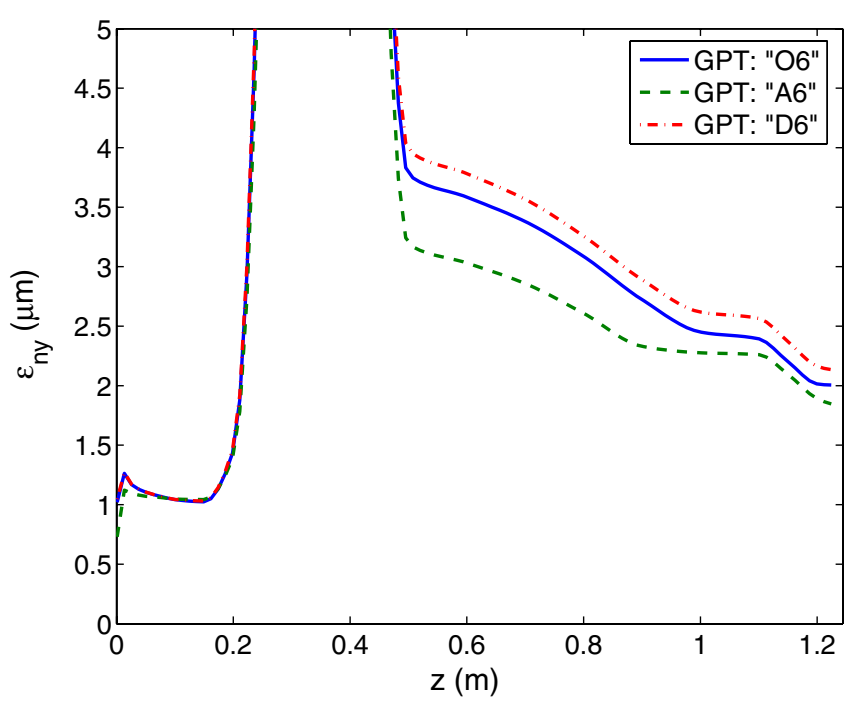

FIG. 10. (Color) Comparison of emittance vs position calculations for GPT with different boundary conditions: open (O6), approximate (A6), and Dirichlet (D6). Refer to the text for details. Bunch charge is $80 \mathrm{pC}$, the solenoid current is $3.6 \mathrm{~A}$, and the gun voltage is $250 \mathrm{kV}$. 100k macroparticles were used in the simulations.

PARMELA3D and GPT. The mesh size was set to $64 \times 64 \times$ 64 for PARMELA3D and $50 \times 50 \times 50$ for the nonequidistant mesh Poisson equation solver in GPT with the bounding box size set to $5 \sigma$ in each dimension. The Poisson equation solver in GPT provides a choice of three different boundary conditions at the bounding box: Dirichlet with zero potential, an open boundary, and an approximate boundary in which the potential at the bounding box is assigned values analytically computed for a uniformly charged brick with rms dimensions equal to those of the actual bunch. Zero potential is assigned at the cathode to include image charge effects for all boundary condition types. Figure 10 shows the emittance vs longitudinal position calculated for the different choices of boundary conditions. Beam envelopes are essentially identical for the different boundary conditions and therefore are not shown. We use the open boundary condition for subsequent comparisons with the measurements.

\section{B. Data processing procedures}

Appropriate noise subtraction is required to extract second moments from the measured beam profiles and phase space distributions. Our approach follows that of the selfconsistent unbiased rms emittance analysis (SCUBEEx) [24], in which: (i) a certain contour separates the signal plus noise region from the noise only region; (ii) the average intensity of the region outside this contour represents the noise bias; (iii) the noise bias is subtracted from the data inside the contour while the outside region is assigned zero intensity; (iv) if only uniform random noise is present, as the contour is enlarged, the parameter of interest should not change significantly once all the signal is accounted for. We have used two types of contours - a circular boundary for the viewscreen data, and a special boundary detection technique for the measured phase space distributions. The boundary detection technique is based on the following observation. A binary image obtained by applying a threshold to measured 2D distribution is likely to form a contiguous region for the signal, and many individual islands for the noise. This is illustrated in Fig. 11 for one of our phase space measurements. The boundary detection algorithm proceeds as follows: (i) the data is convoluted with a uniform $n \times n$ square (image blurring); (ii) the smallest threshold is found that generates a single continuous island; (iii) $n$ is incremented and step (i) is repeated. The process stops when the island begins to include clearly visible chunks of the noise region. Once the boundary has been found, noise subtraction is verified by growing and shrinking the contour. The contour growth is stopped when less than half of the entire image area is available for noise estimation. Figure 12 illustrates the procedure further. The
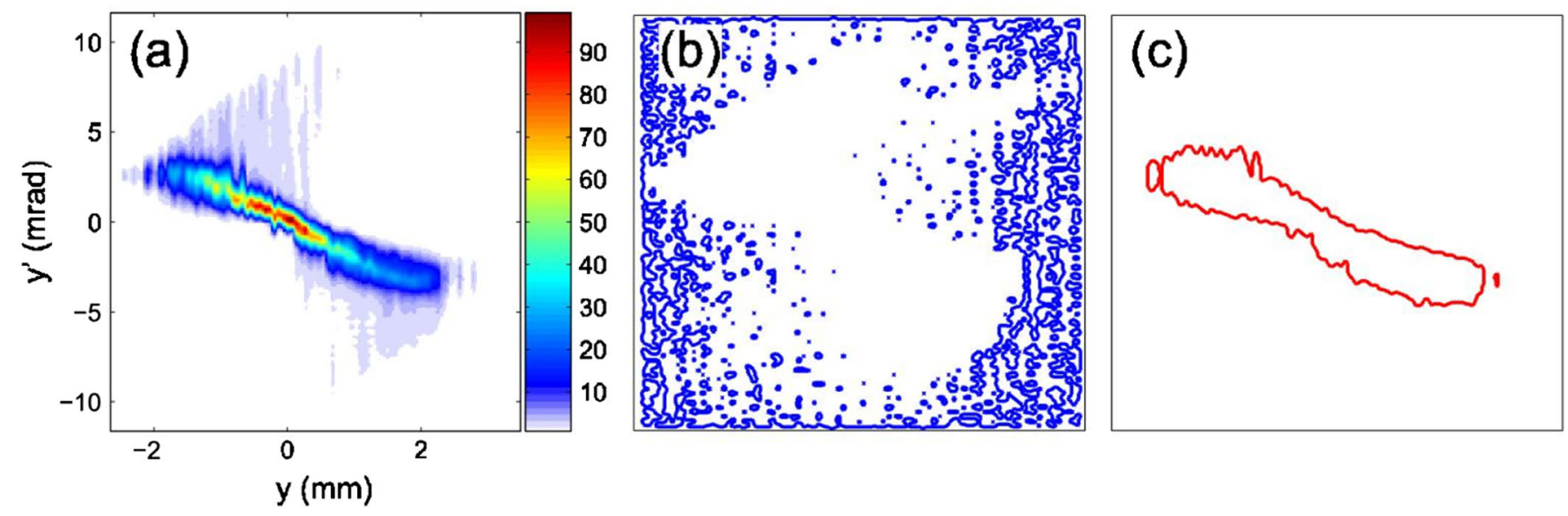

FIG. 11. (Color) Example of measured transverse phase space (a), with a contour map of a binary image after applying $0.6 \%$ (b) and $4.4 \%$ (c) threshold of the peak intensity. 

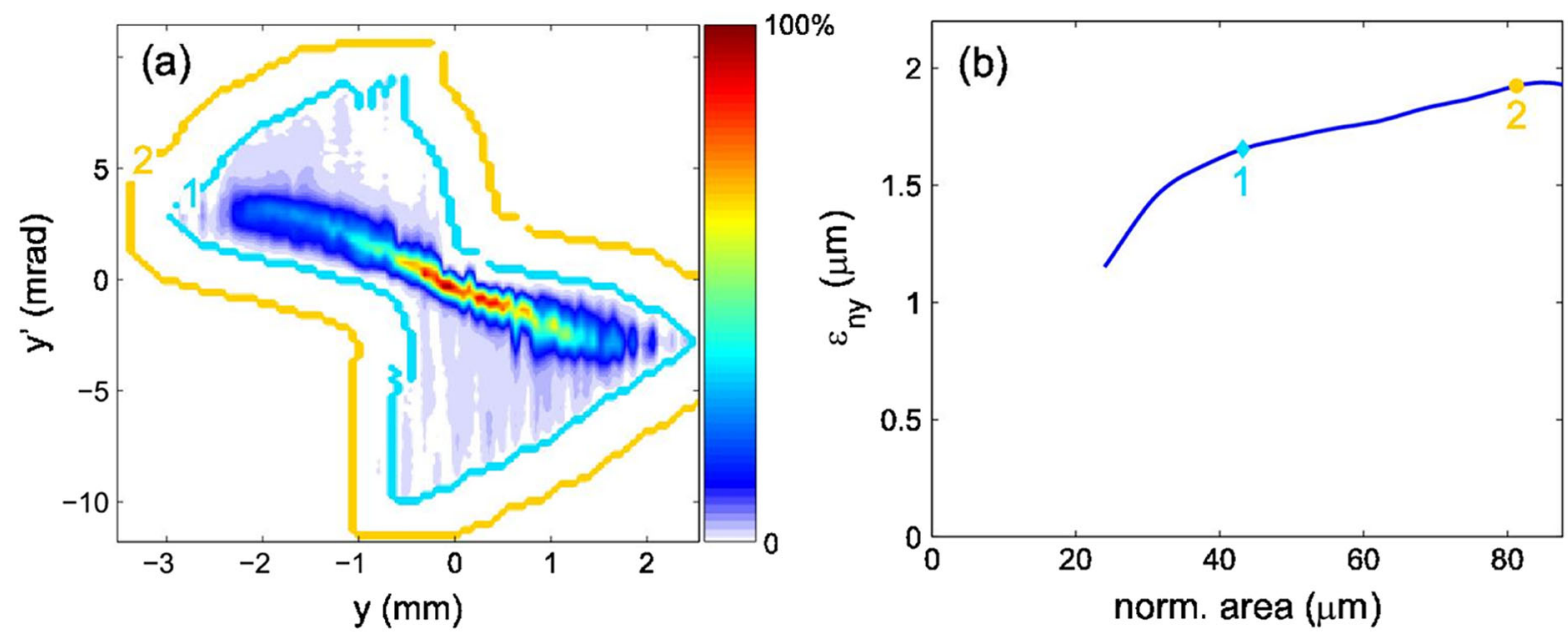

FIG. 12. (Color) Noise subtraction verification procedure. (a) Example transverse phase space with the contour (1) obtained through the boundary detection algorithm and the grown contour (2), which corresponds to $50 \%$ of the available data treated as noise. (b) Normalized rms emittance calculation as a function of included area after the noise subtraction. Refer to the text for details.

change in the parameter of interest-the rms emittance in this case-with the area included within the boundary separating the signal and noise regions represents the uncertainty in the measurement due to the noise subtraction. Emittance or rms values calculated this way correspond to $100 \%$ of the beam.

\section{COMPARISON OF MEASUREMENTS WITH SIMULATIONS}

\section{A. Transverse phase space distributions}

Figure 13 shows the comparison of the measured transverse phase space at the location of the $1 \mathrm{st}$ slit for $0.5 \mathrm{pC}$ charge per bunch with PARMELA3D and GPT simulations. The resolution of the measured transverse phase space is $90 \times 90$ steps. For the simulations, each image is produced from a $300 \times 3002 \mathrm{D}$ histogram with additional convolution with a uniform $3 \times 3$ kernel. Each image is normal- ized to the same maximum intensity value. A color map identical to that of Fig. 6 is used throughout. As expected, the calculated rms normalized emittance is in good agreement with the thermal emittance value for this case.

Figures 14 and 15 show the measured and simulated transverse phase space for the 20 and $80 \mathrm{pC}$ bunch charge cases, respectively, as a function of the solenoid lens strength. The streak features seen in the measured phase space are due to the motion of the laser spot at the photocathode. Good qualitative agreement is seen for $20 \mathrm{pC} /$ bunch data, while some discrepancy in the shape of transverse phase space distributions can be seen in the $80 \mathrm{pC} /$ bunch case, particularly at larger solenoid current values.

\section{B. Second moments of the beam}

Figure 16 shows a comparison of the vertical rms beam size (a) at the location of the viewscreen $(z=1.244 \mathrm{~m})$
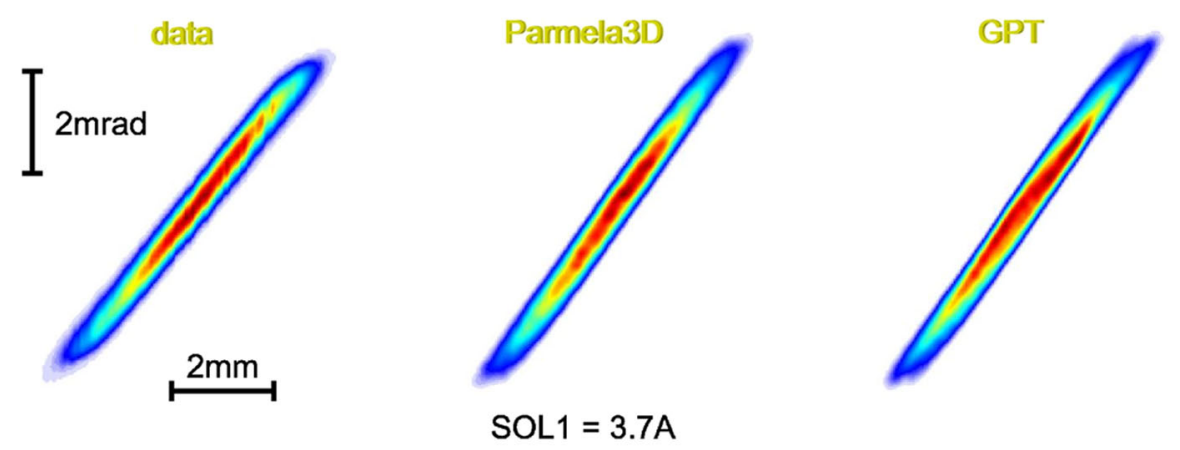

FIG. 13. (Color) Comparison of measured and simulated vertical transverse phase space distributions for $0.5 \mathrm{pC}$ bunches at $z=$ $1.244 \mathrm{~m}$. Solenoid current is $3.7 \mathrm{~A}$. Corresponding rms normalized emittances: $\epsilon_{n, y}=0.31 \pm 0.04 \mu \mathrm{m}$ (data), $0.29 \mu \mathrm{m}$ (PARMELA3D), $0.28 \mu \mathrm{m}$ (GPT). Corresponding rms sizes: $\sigma_{y}=1.15 \pm 0.05 \mathrm{~mm}$ (data), $1.14 \mathrm{~mm}$ (PARMELA3D), 1.14 (GPT). 


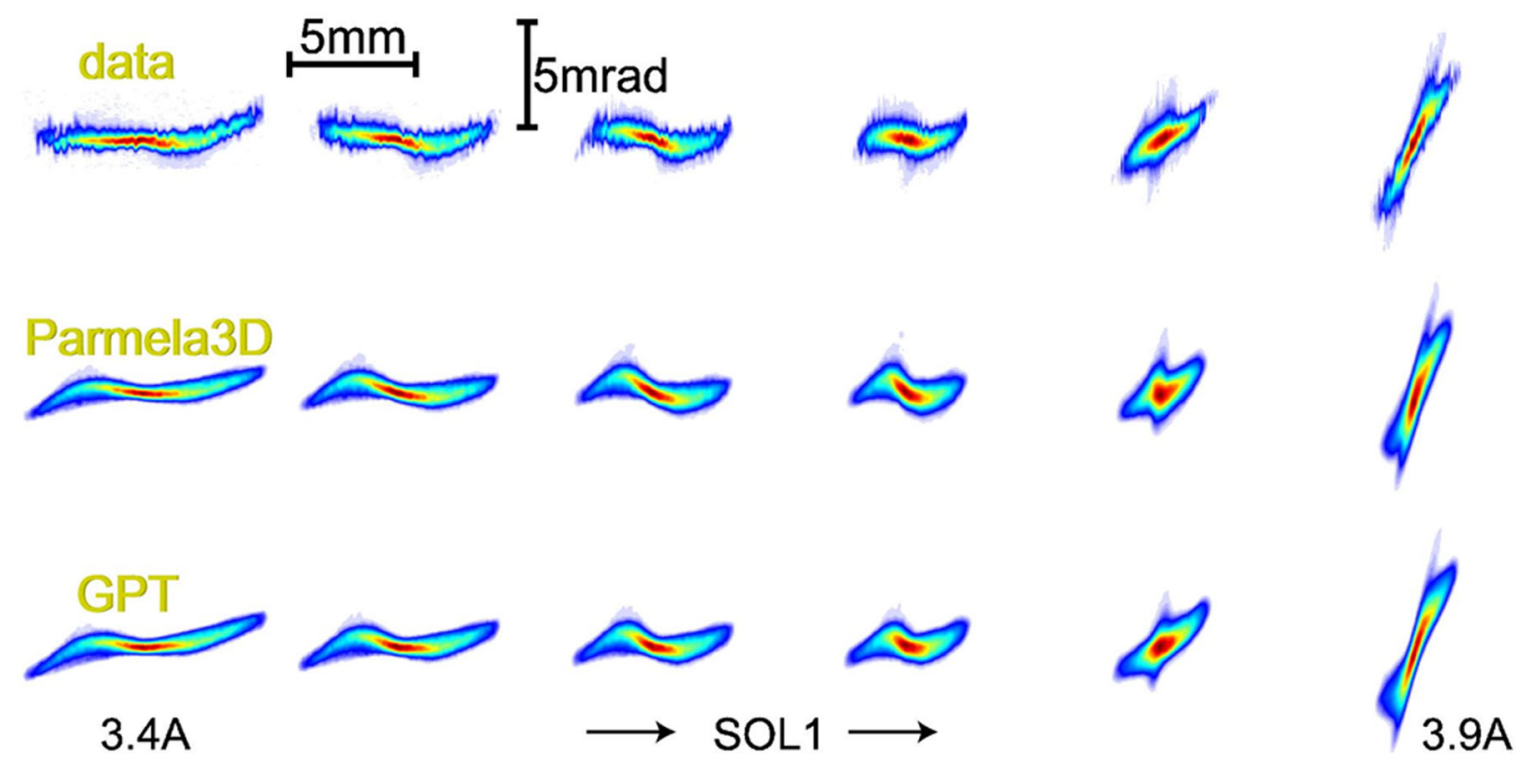

FIG. 14. (Color) Comparison of measured and simulated vertical transverse phase space distributions for $20 \mathrm{pC}$ bunches at $z=$ $1.244 \mathrm{~m}$. Data representing measurements, PARMELA3D, and GPT calculations are arranged in rows with different strength of the solenoid lens corresponding to column position.

and rms normalized vertical emittance for $100 \%$ of the beam (b) as a function of solenoid current for $20 \mathrm{pC}$ bunches. Excellent agreement is seen for the spot-size comparison and good overall agreement for the emittance values, although the measured rms emittance appears to be systematically smaller for the $20 \mathrm{pC} /$ bunch case.
Figure 17 shows similar results for $80 \mathrm{pC}$ charge bunches. Different sets of curves for EMS and viewscreen simulations correspond to different laser spots as registered for the two data sets. Good agreement between simulations and measurements of the beam size is seen for $80 \mathrm{pC} /$ bunch before the formation of a beam waist at the
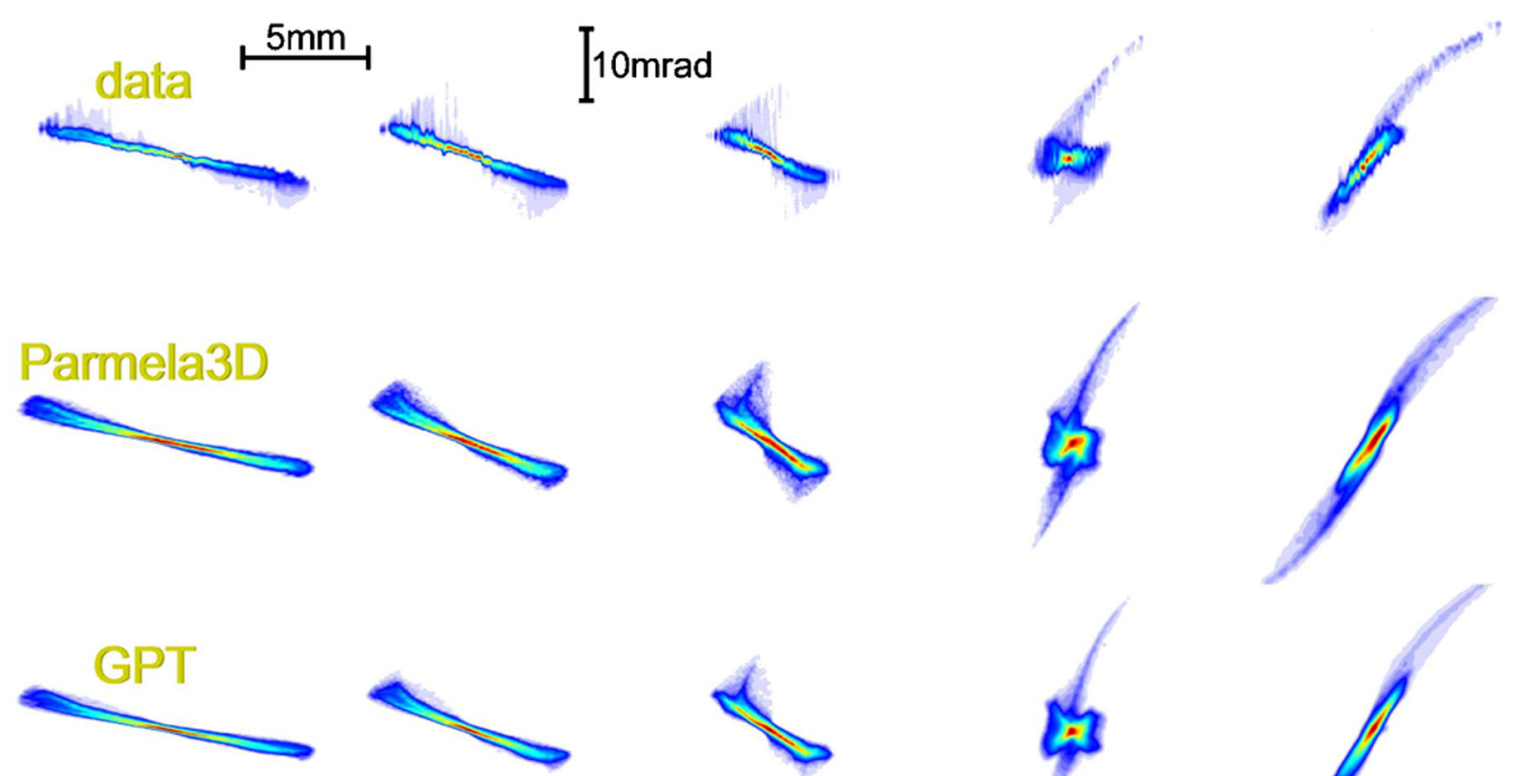

$3.4 \mathrm{~A}$
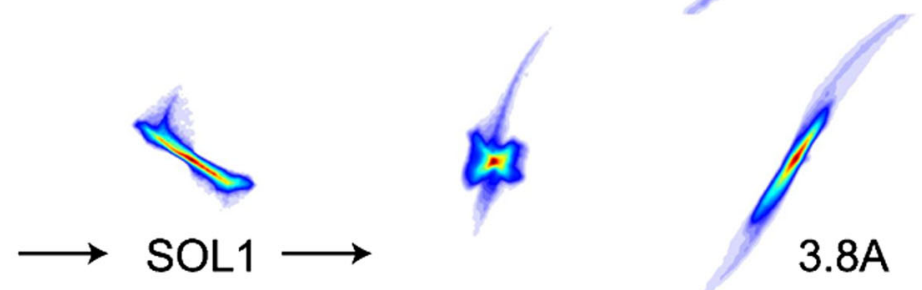

FIG. 15. (Color) Comparison of measured and simulated vertical transverse phase space distributions for $80 \mathrm{pC}$ bunches at $z=$ $1.244 \mathrm{~m}$. Data representing measurements, PARMELA3D, and GPT calculations are arranged in rows with different strength of the solenoid lens corresponding to column position. 

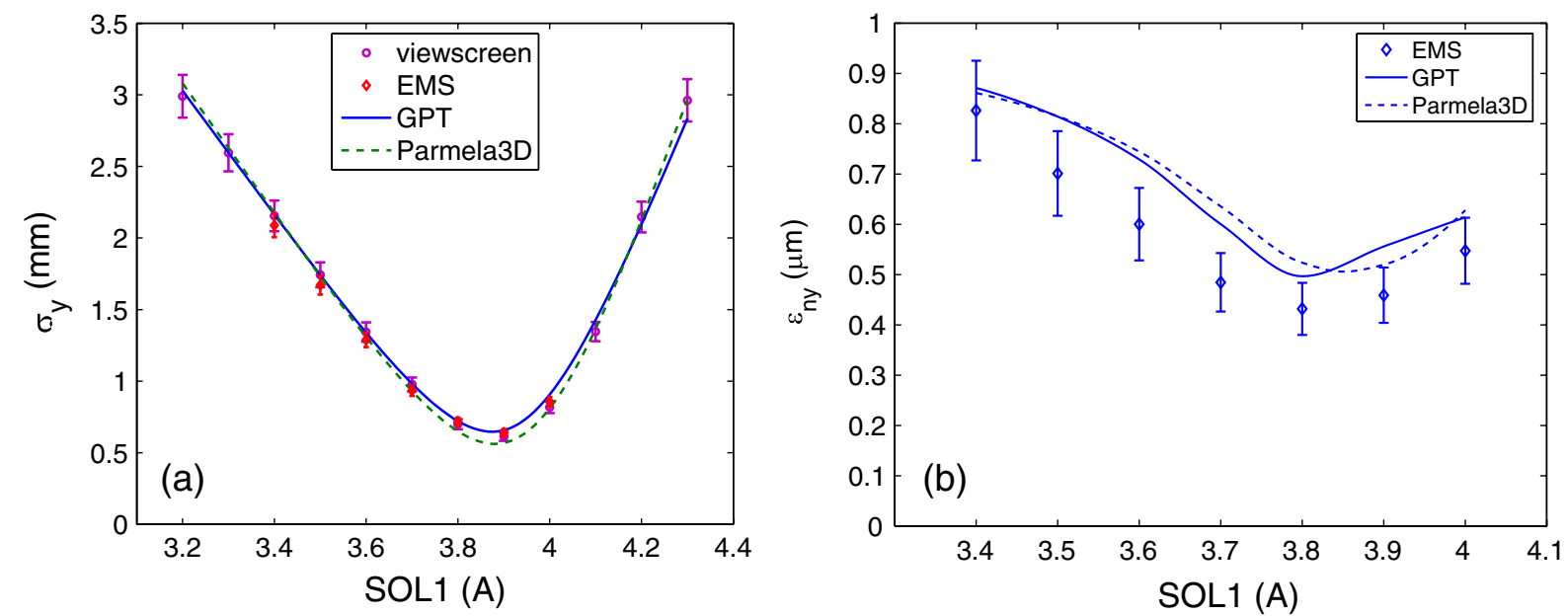

FIG. 16. (Color) Comparison of vertical rms beam size (a) and normalized rms emittance (b) at the location of the measurement $z=1.244 \mathrm{~m}$ vs solenoid excitation current for $20 \mathrm{pC}$ bunches.

location of measurement, while the agreement at larger solenoid currents is less conclusive.

\section{Asymmetric phase space distributions}

Asymmetric phase space distributions have been measured on multiple occasions for space charge dominated beam conditions in our setup. For example, see Fig. 18. No such asymmetry was observed for low bunch charge running under otherwise identical operating conditions. It was important to understand the origin of this behavior for its subsequent mitigation. We were able to reproduce similar phase space distributions in 3D simulations for laser spots with noticeable asymmetry. To understand the mechanism for this tail formation, the particles comprising the tail have been tagged and their position in the transverse profile distributions at the location of the photocathode and inside the solenoid are shown in Fig. 19. The asymmetry in the

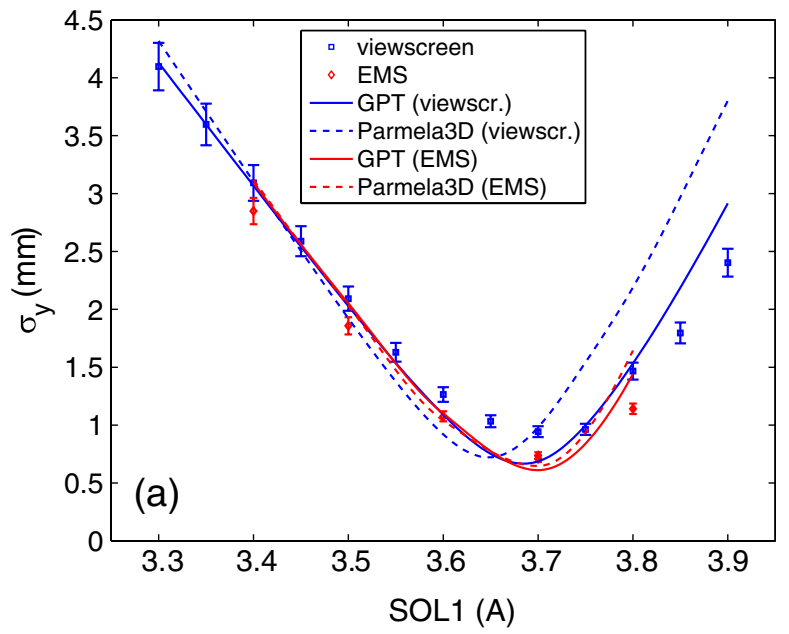

laser spot causes the space charge forces to push the tail particles further from the solenoid axis [there is about $2 \mathrm{~mm}$ difference between the distances from the center to the top and bottom edges of the transverse distribution in Fig. 19(c)] where they experience a stronger kick from the solenoid lens. These particles then undergo a crossover and form the observed phase space tail. The rms normalized emittance is increased from 1.8 to $2.6 \mu \mathrm{m}$ as a result. Improving the transverse laser shape would reduce the asymmetry in the phase space.

\section{Theoretical limit to beam brightness}

It is instructive to consider rms emittance as a function of the contributing beam fraction [19]. For example, Fig. 20 shows the rms normalized emittance vs beam fraction as measured and simulated using GPT for $20 \mathrm{pC} /$ bunch for a solenoid current of 3.8 A. Figure 21

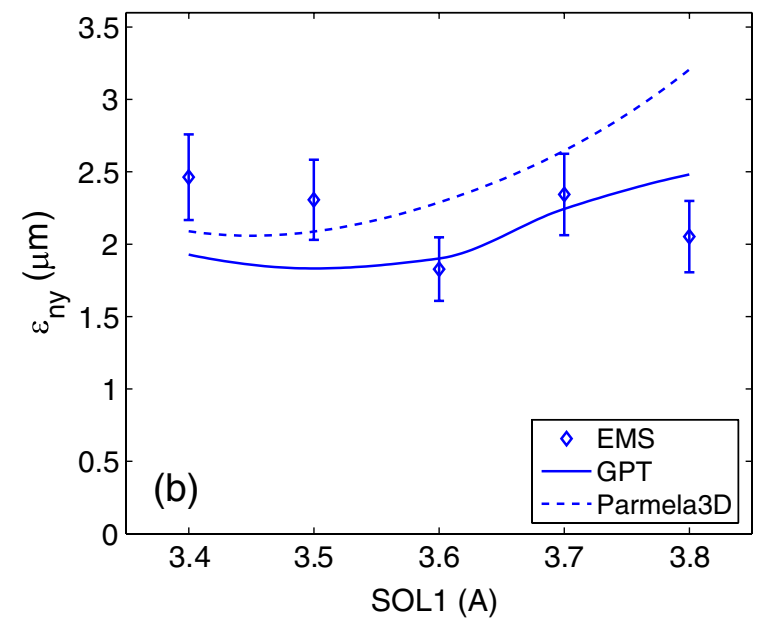

FIG. 17. (Color) Comparison of vertical rms beam size (a) and normalized rms emittance (b) at the location of the measurement $z=1.244 \mathrm{~m}$ vs solenoid excitation current for $80 \mathrm{pC}$ bunches. 


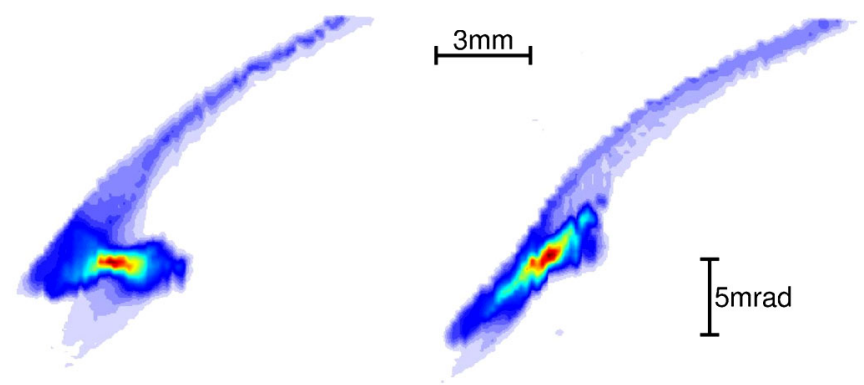

FIG. 18. (Color) Example of asymmetric phase space distribution measured for $80 \mathrm{pC} /$ bunch at the solenoid current of $3.7 \mathrm{~A}$ (left) and 3.8 A (right). The top part of the asymmetric tail was truncated during the measurement.

shows corresponding results for $80 \mathrm{pC} /$ bunch for a solenoid current of 3.6 A. Dot-dashed lines show the expected curve for a 2D Gaussian distribution in the phase space with the same rms emittance as $100 \%$ of the actual beam. It can be seen that, in the case of $80 \mathrm{pC} /$ bunch, the beam strongly deviates from Gaussian distribution, having a substantially brighter core. The core emittance, defined as $\epsilon_{n, y, \text { core }} \equiv d \epsilon_{n, y}(\xi=0) / d \xi$ with $\epsilon_{n, y}(\xi)$ being the normalized rms emittance as a function of beam fraction $0 \leq$ $\xi \leq 1$, is given in both figures along with the core fraction $\xi_{\text {core }}$ defined as the fraction of the beam with the emittance equal to the core emittance value: $\epsilon_{n, y}\left(\xi_{\text {core }}\right)=\epsilon_{n, y, \text { core }}$.

The beam brightness available from photoinjectors forms through an interplay of phenomena such as space charge dominated beam dynamics in the presence of time transient and position dependent external fields. The upper limit, however, is set by the thermal emittance of the photocathode and the available accelerating gradient.

Consider a short laser pulse illuminating a photocathode placed in the accelerating field $E_{\text {cath }}$. The electron bunch after the emission will assume a pancake shape provided that the laser pulse duration is sufficiently short: $\sigma_{t} \lesssim$ $\sqrt{\sigma_{\perp} m / e E_{\text {cath }}}, m$ and $e$ are the electron mass and charge, respectively. This condition is satisfied in most operating photoinjectors delivering beams with short duration bunches. The maximum charge density that can be supported by the electric field is then given approximately by

$$
\frac{d q}{d A} \approx \epsilon_{0} E_{\text {cath }} .
$$

The image charge is included in the equation above. The average (normalized) beam brightness can be defined as the ratio of the average current $I_{\text {avg }}$ over its $4 \mathrm{D}$ volume $\mathcal{A}_{4}$ defined for $\left(x, p_{x} / m c, y, p_{y} / m c\right)$ coordinates:

$$
\mathcal{B}_{n, \text { avg }}=\frac{I_{\text {avg }}}{\mathcal{A}_{4}} .
$$

Beam brightness normalized per single bunch is given by $\mathcal{B}_{n \text {,avg }} / f=q / \mathcal{A}_{4}$, with $f$ being the repetition rate, and $q$ the charge contained in the 4D volume $\mathcal{A}_{4}$. For example, the $4 \mathrm{D}$ volume $\mathcal{A}_{4}=d x d p_{x} d y d p_{y} /(m c)^{2}$ for a $4 \mathrm{D}$ hypercuboid element with sides $d x, d p_{x}, d y$, and $d p_{y}$. The charge contained in $\mathcal{A}_{4}$ can be written as

$$
\frac{d q}{d A} d x d p_{x} d y d p_{y} \frac{1}{\kappa \sigma_{p}^{2}},
$$

with $\sigma_{p}$ being the rms value of the transverse momentum (assumed to be isotropic for both transverse directions), which is $\sigma_{p}=\sqrt{m k T_{\perp}}$ for the Maxwell-Boltzmann distribution of velocities. The dimensionless coefficient $\kappa$ depends on the details of the momentum distribution, e.g. $\kappa=4 \pi$ corresponds to a uniform circular distribution in $p_{x}$ and $p_{y}$ with a diameter $4 \sigma_{p}$, while $\kappa=2 \pi$ corresponds to the peak of a 2D Gaussian distribution. Combining Eq. (4) with the charge density as given by Eq. (2), we find the maximum beam brightness normalized per single
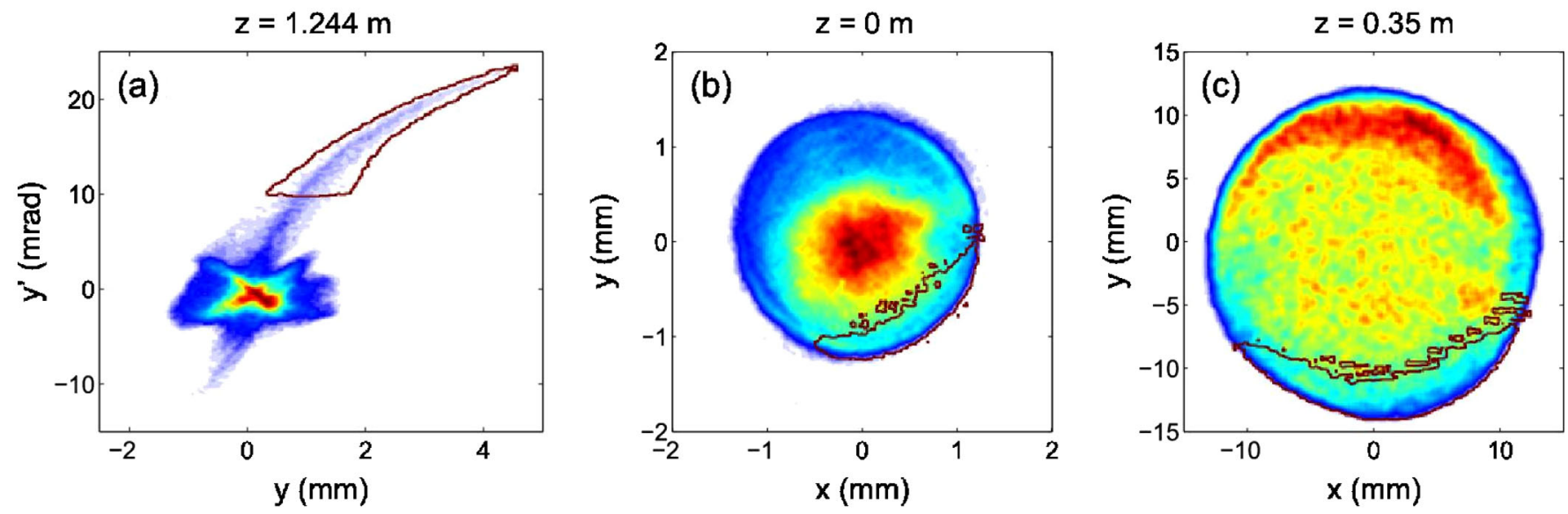

FIG. 19. (Color) Calculated vertical transverse phase space using GPT for $80 \mathrm{pC} /$ bunch (a) at the location of the $1 \mathrm{st}$ slit $(z=1.244 \mathrm{~m}$ ) for a given transverse initial laser spot size (b). Transverse profile is shown at the location inside the solenoid (c), $z=0.35 \mathrm{~m}$. Note two different scales for (b) and (c). The portion of the distribution responsible for the tail is also shown. 

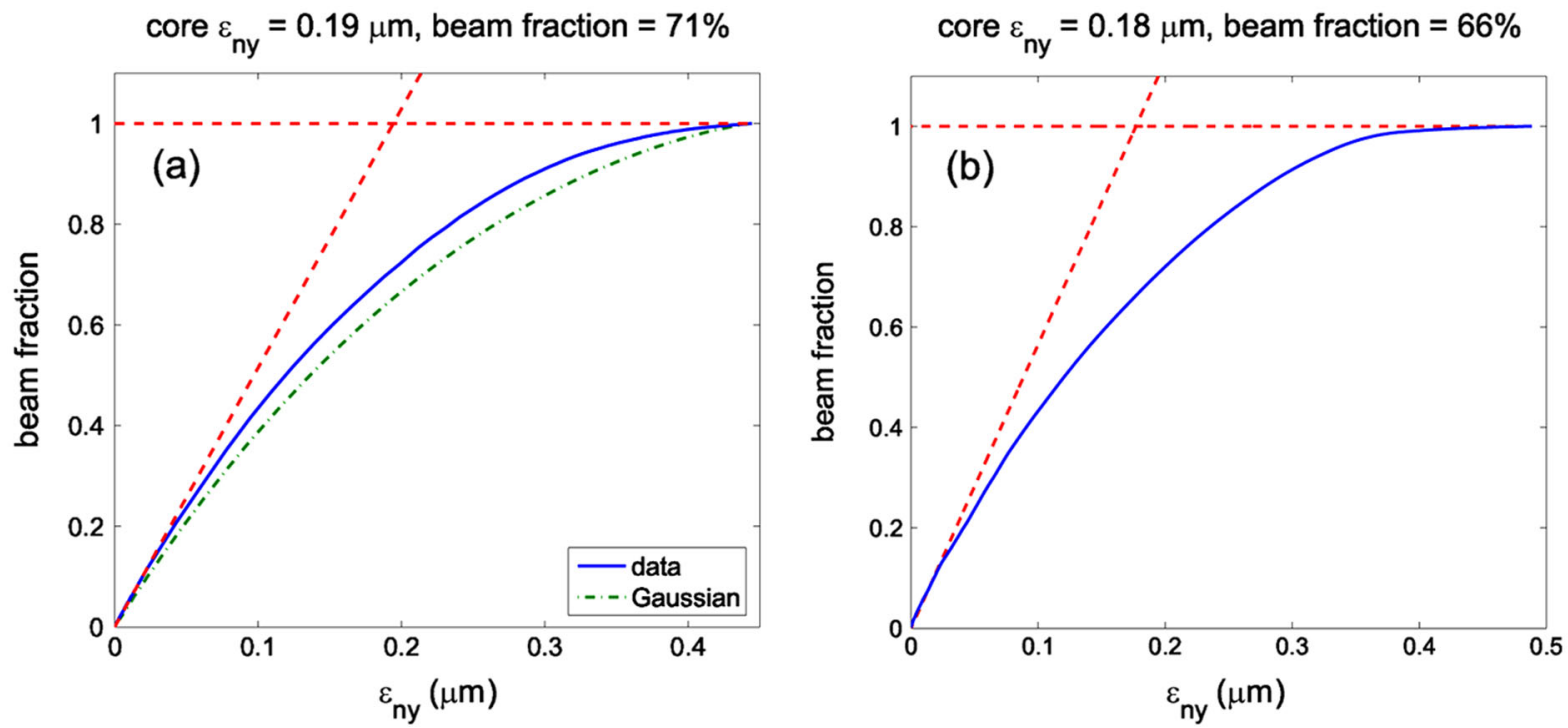

FIG. 20. (Color) Normalized rms emittance vs included beam fraction for measured (a) and calculated by GPT (b) phase space distributions for $20 \mathrm{pC} /$ bunch. A corresponding Gaussian beam is also shown for comparison. The $100 \%$ normalized rms emittance is $0.43 \pm 0.05 \mu \mathrm{m}$ for the measurement (a) and $0.49 \mu \mathrm{m}$ for GPT (b).

bunch

$$
\frac{\mathcal{B}_{n, \mathrm{avg}}}{f}=\frac{\epsilon_{0} m c^{2}}{\kappa} \frac{E_{\mathrm{cath}}}{k T_{\perp}} .
$$

To compare Eq. (5) with the measured data, we compute the beam brightness per single bunch as

$$
\left.\frac{\mathcal{B}_{n, \text { avg }}}{f}\right|_{\text {meas }}=q\left(\frac{\xi}{4 \pi \epsilon_{n, y}(\xi)}\right)^{2},
$$

where $q$ is the full bunch charge, and $\epsilon_{n, y}(\xi)$ is the emit-

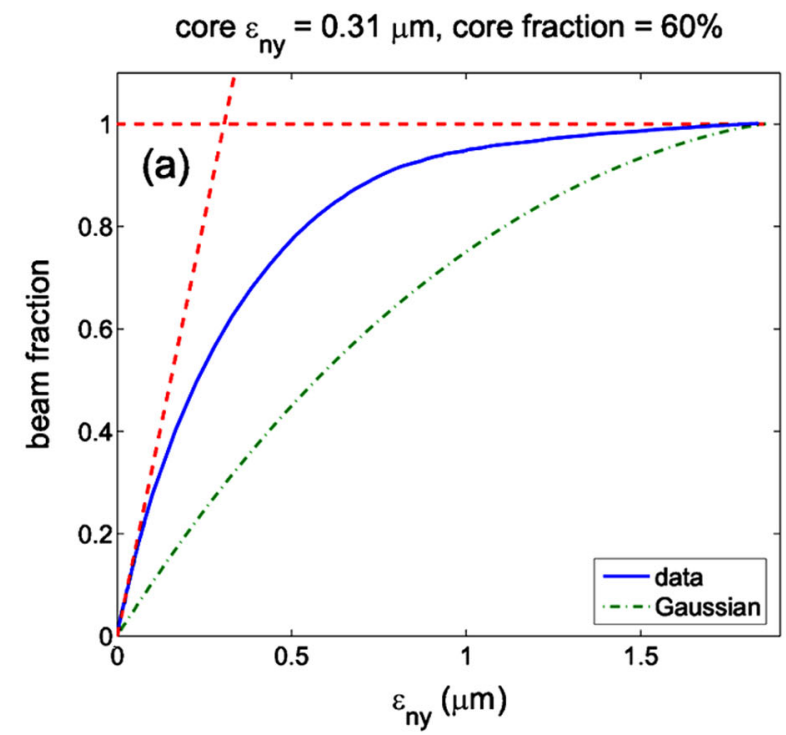

tance vs beam fraction curve (cf. Fig. 21). Equation (6) assumes an axially symmetric beam with a uniformly populated phase space distribution inside an equivalent ellipse with $4 \pi \epsilon_{n, y}(\xi)$ area. Figure 22 shows the brightness normalized per single bunch vs the beam fraction. The theoretical maximum brightness as given by Eq. (5) is shown as well $(\kappa=4 \pi$ and $2 \pi)$. Additionally, $\mathcal{B}_{n \text {,avg }} / f$ is computed for a beam that has the same $100 \% \mathrm{rms}$ emittance as the actual beam but instead adopts a 2D Gaussian distribution in the phase space. It is seen that

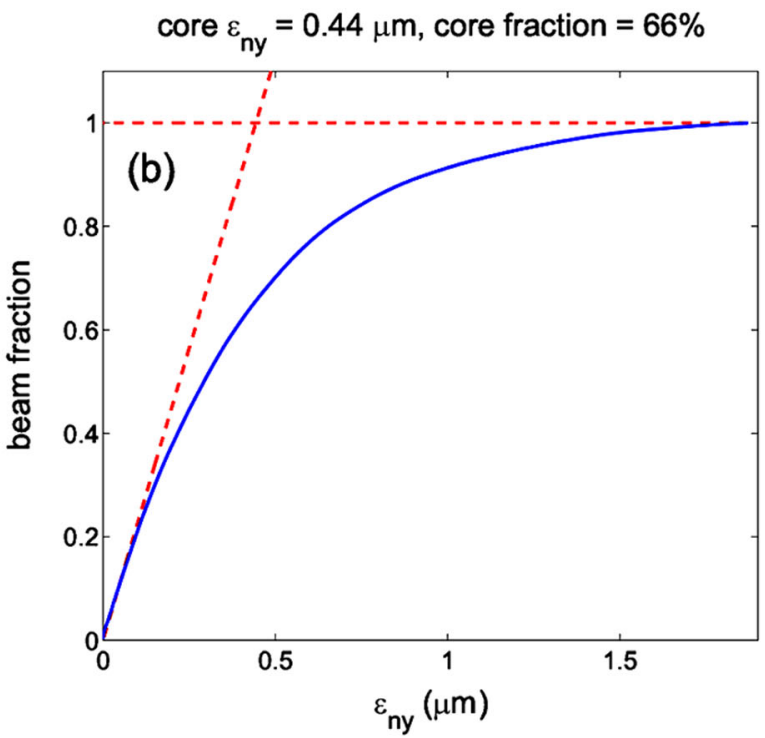

FIG. 21. (Color) Normalized rms emittance vs included beam fraction for measured (a) and calculated by GPT (b) phase space distributions for $80 \mathrm{pC} /$ bunch. A corresponding Gaussian beam is also shown for comparison. The $100 \%$ normalized rms emittance is $1.8 \pm 0.2 \mu \mathrm{m}$ for the measurement (a) and $1.8 \mu \mathrm{m}$ for GPT (b). 


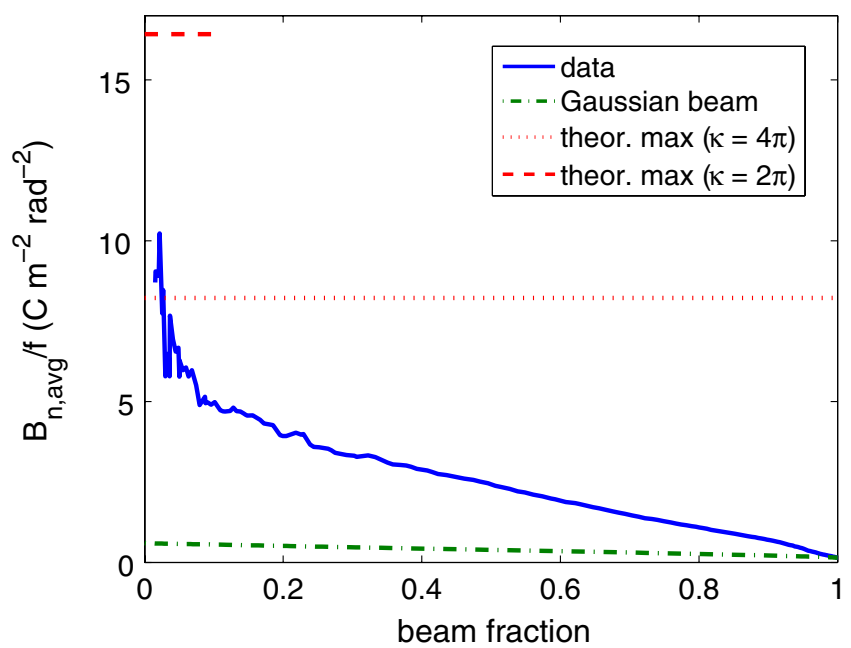

FIG. 22. (Color) Beam brightness normalized per single bunch as a function of contributing beam fraction for $80 \mathrm{pC}$ charge per bunch. Corresponding result for an equivalent Gaussian beam with the same $100 \%$ rms emittance is shown for comparison (dot-dashed line). Dotted and dashed lines show the theoretical limit as given by Eq. (5) for $\kappa=4 \pi$ and $2 \pi$, respectively. The rms normalized emittance is $1.8 \pm 0.2 \mu \mathrm{m}$.

an equivalent Gaussian beam does a poor job of describing the $80 \mathrm{pC} /$ bunch beam as the core is substantially brighter for the measured beam approaching the limit given by Eq. (5). For a 2D Gaussian distribution in the transverse momentum of photoemitted electrons $(\kappa=2 \pi)$, the measured core brightness of the beam is about a factor of 2 lower than the limit given by Eq. (5).

\section{DISCUSSION AND OUTLOOK}

The benchmarking of 3D space charge codes has been performed with the direct measurements of the transverse phase space for a bunched beam in the space charge dominated regime from a dc photoemission gun. Overall, good agreement has been found between the measurements and simulations. We observe that an equivalent Gaussian beam assigned the measured $100 \%$ rms emittance poorly describes the peak brightness of the beam at $80 \mathrm{pC}$ bunch charge, due to the presence of a substantially brighter core. In particular, for the case of a measured $100 \%$ rms normalized emittance of $\epsilon_{n, y}=1.8 \pm 0.2 \mu \mathrm{m}$, the core emittance is found to be $\epsilon_{n, y, \text { core }}=0.31 \pm 0.04 \mu \mathrm{m}$ with $60 \%$ beam fraction contained in the core. Furthermore, comparison of the measured beam brightness vs beam fraction shows that it approaches within a factor of 2 the maximum theoretical brightness as set by the available accelerating gradient and transverse thermal energy of the photocathode. While the maximum brightness of the beam cannot be improved without changing $k T_{\perp}$ and $E_{\text {cath }}$, it should be possible to bring a larger fraction of the electron bunch charge within the brightness limit given by Eq. (5) through proper control of the space charge forces. For example,

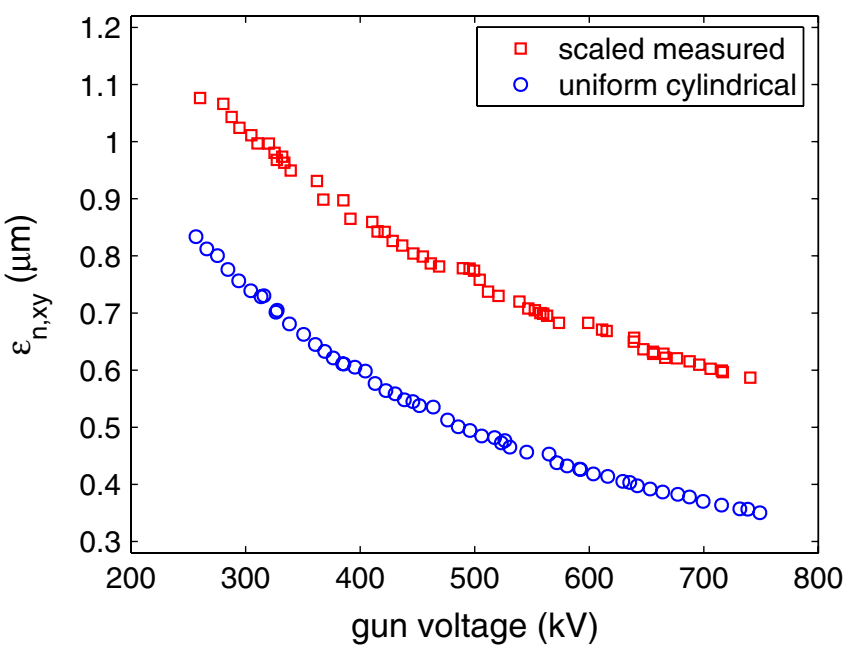

FIG. 23. (Color) Calculated normalized rms emittance (100\% of the beam) at $z=1.244 \mathrm{~m}$ vs the gun voltage for the beam line setup used in this study. Refer to the text for details on simulation parameters.

Fig. 23 shows the simulated possible rms normalized emittance ( $100 \%$ of the beam) from the same beam line as used in this experiment for $80 \mathrm{pC}$ bunches as a function of gun voltage. Two different distributions have been used in these calculations: a scaled distribution created from the measured laser transverse and temporal profiles, and a uniform cylindrical laser distribution. The same thermal transverse energy was used corresponding to GaAs illuminated by $520 \mathrm{~nm}$. The laser pulse duration in these simulations was 12 ps rms, about 50\% longer than that was used in the measurements reported here. Similarly, the laser spot size on the photocathode was about 50\% larger in these simulations than in the measurements for the $250 \mathrm{kV}$ case. These simulations were done using 20k macroparticles representing the bunch. In addition to employing a more optimal laser pulse duration and size and continuing the work to reach the gun design voltage of $750 \mathrm{kV}$, noticeable reduction in emittance can be achieved by improving the laser pulse shape and the pointing stability.

Finally, we note that despite the significantly more complicated setup and beam dynamics in the full ERL injector [25], where the bunch undergoes acceleration to over $10 \mathrm{MeV}$, bunch compression and matching into the linac, a much simpler beam line such as the one used in this study allows exploration of the best beam brightness achievable from the complete photoinjector. More specifically, simulations for the full injector [26], where substantially shorter bunches are produced though subsequent drift bunching ( $\sim 3 \mathrm{ps} \mathrm{rms})$, indicate rms normalized emittances at $\sim 11 \mathrm{MeV}$ which are about $50 \%$ lower but otherwise very similar to those shown in Fig. 23. Thus, to continue the work on improving the HV dc gun design and pushing for lower emittances, it is sufficient in many ways to have a simpler setup with beam diagnostics dedicated to such research similar to the one described in this work. 


\section{ACKNOWLEDGMENTS}

We acknowledge control system support by John Dobbins, John Barley, and Mike Forster, laser support by Dimitre Ouzounov and Heng Li, magnetic measurements by Alexander Temnykh, and mechanical engineering support by Karl Smolenski. Tsukasa Miyajima, Georg Hoffstaetter, and Dave Rice are acknowledged for many useful discussions and their interest in this work. This work is supported by the NSF Grant No. PHY-0131508 and NSF/ NIH-NIGMS Award No. DMR-0225180.

[1] J. Billen and L. Young, Los Alamos Laboratory Technical Report No. LA-UR-96-1835, 1996 (revised 2005).

[2] K. Flottmann, Astra-A Space Charge Tracking Algorithm, available at http://www.desy.de/ mpyflo.

[3] GPT-general particle tracer, Pulsar Physics, http:// www.pulsar.nl/gpt.

[4] M. Ferrario, J.E. Clendenin, D. T. Palmer, J. B. Rosenzweig, and L. Serafini, Report No. SLAC-PUB8400, 2000.

[5] L. Giannessi and M. Quattromini, Phys. Rev. ST Accel. Beams 6, 120101 (2003).

[6] D. P. Rusthoi and K. R. Crandall, Los Alamos Laboratory Technical Report No. LA-UR-97-886, 1997.

[7] MAFIA, CST GmbH, Darmstadt, Germany, http:// www.cst.de.

[8] D. P. Grote, A. Friedman, and I. Haber, in Proceedings of the 1996 Computational Accelerator Physics Conference, AIP Conf. Proc. No. 391 (AIP, Williamsburg, VA, 1996), p. 51.

[9] A. Cianchi et al., Phys. Rev. ST Accel. Beams 11, 032801 (2008).

[10] V. Miltchev et al., in Proceedings of the 2004 FEL Conference (EPS-AG and CERN, Trieste, Italy, 2004), p. 399.

[11] Mini-Workshop On Characterization Of High Brightness Beams (DESY, Zeuthen, Germany, 2008), https://indico. desy.de/conferenceDisplay.py?confId=806.

[12] B. M. Dunham, C. K. Sinclair, I. V. Bazarov, Y. Li, X. Liu, and K. W. Smolenski, in Proceedings of the 2007 Particle
Accelerator Conference, Albuquerque, New Mexico, 2007 (IEEE, Albuquerque, New Mexico, 2007), p. 1224.

[13] J. Billen and L. Young, Los Alamos Laboratory Technical Report No. LA-UR-96-1834, 2000.

[14] Belomestnykh, V. Shemelin, K. Smolenski, and V. Veshcherevich, in Proceedings of the 2007 Particle Accelerator Conference, Albuquerque, New Mexico, 2007, Ref. [12], p. 2331.

[15] D. G. Ouzounov, I. V. Bazarov, B. M. Dunham, C. K. Sinclair, S. Zhou, and F. Wise, in Proceedings of the 2007 Particle Accelerator Conference, Albuquerque, New Mexico, 2007, Ref. [12], p. 530.

[16] S. Agostinelli et al. (GEANT4 Collaboration), Nucl. Instrum. Methods Phys. Res., Sect. A 506, 250 (2003).

[17] C. Gulliford, Cornell University, Internal Report, 2006, available at http://www.lepp.cornell.edu/ ib38/reu/06/ gulliford.pdf.

[18] S. G. Anderson, J. B. Rosenzweig, G. P. Le Sage, and J. K. Crane, Phys. Rev. ST Accel. Beams 5, 014201 (2002).

[19] C. Lejeune and J. Aubert, Adv. Electron. Electron Phys. Suppl. A 13, 159 (1980).

[20] I. V. Bazarov, D. G. Ouzounov, B. M. Dunham, Y. Li, X. Liu, R. E. Meller, J. Sikora, C. K. Sinclair, F. W. Wise, and T. Miyajima, Phys. Rev. ST Accel. Beams 11, 040702 (2008).

[21] I. V. Bazarov, B. M. Dunham, Y. Li, X. Liu, D. G. Ouzounov, C. K. Sinclair, F. Hannon, and T. Miyajima, J. Appl. Phys. 103, 054901 (2008).

[22] L. M. Young and J. Billen, in Proceedings of the Particle Accelerator Conference, Portland, OR, 2003 (IEEE, New York, 2003).

[23] S. B. van der Geer, O. J. Luiten, M. J. de Loos, G. Poplau, and U. van Rienen, Report No. DESY-TESLA-2003-04, 2003.

[24] M.P. Stockli, R.F. Welton, and R. Keller, Rev. Sci. Instrum. 75, 1646 (2004).

[25] I. V. Bazarov and C. K. Sinclair, Phys. Rev. ST Accel. Beams 8, 034202 (2005).

[26] I. V. Bazarov, B. M. Dunham, F. Hannon, Y. Li, X. Liu, T. Miyajima, D. G. Ouzounov, and C.K. Sinclair, in Proceedings of the 2007 Particle Accelerator Conference, Albuquerque, New Mexico, 2007, Ref. [12], p. 1221. 